$\xi=-1$

\title{
A Novel Approach for the Detection, Classification and Localization of Transmission Lines Faults using Wavelet Transform and Support Vector Machines Classifier
}

\author{
Nilish S.Wani ${ }^{1 *}$, Dr. R. P. Singh ${ }^{2}$ \\ ${ }^{1}$ Research Scholar, Department of Electrical Engineering, Sri SatyaSai University of Technology \& Medical Sciences, \\ Pachama, Sehore, (M.P.), India. \\ ${ }^{2}$ Professor, Sri SatyaSai University of Technology \& Medical Sciences, Pachama, Sehore, (M.P.), India. \\ *Email: nileshswani@gmail.com
}

\begin{abstract}
Over the time, techniques have been developed whose approach has been linked mainly to the diagnosis of faults, which is why over the years these techniques have been improved little by little in order to complement or at best cases innovate the traditional methods used for the detection of faults from the mathematical point of view relying mainly on sophisticated methods and some of them related to artificial intelligence. Taking into account the aforementioned, in this paper we propose the use one of the branches of artificial intelligence, specifically automatic learning through the tool known as Support Vector Machines (SVM) to find a method with which is feasible to identify and classify the type of fault. For the creation of the mathematical model it is essential to have a database. The database consists of input data and output data, the input data are the detail coefficients obtained from the decomposition of the current and voltage signals using the Discrete Wavelet Transform (DWT). Meanwhile, the output data are the labels assigned and with which the model can identify and classify the different types of faults. Both current signals and voltage signals are generated based on an extensive simulation of faults along the longest transmission line that has a test system.
\end{abstract}

Keywords:DWT; EPS; SVM; Transmission Line Faults.

\section{Introduction}

Taking into account the continuous growth of energy demand worldwide, it is necessary to comply with two very important requirements: The continuity and quality of the electric power supply, these requirements will guarantee the proper functioning of the Electric Power System [1]. The continuity is related to the fact that the Power Electric System must guarantee that the energy generated in the different generation plants is transported long distances uninterruptedly to finally be distributed to the main consumption centers. On the other hand, the quality of electric power could be defined as a set of rules, the same ones that establish levels of voltage, current or frequency that guarantee the useful life, both of electrical and electronic devices that are normally connected to the net. It is practically impossible to rule out the existence of faults in any of the elements that make up the SEP, for this purpose a system of protections must be considered in the previous design, the same one that aims to minimize the effects of the different faults that may occur in the system. The correct performance of the protection system guarantees compliance with the two requirements mentioned above, ensuring that, in the event of a fault in the system, both suffer as little as possible for a short period of time. There are electrical equipment such as generators and power transformers that are not exposed to the weather, they are generally located within generation plants, so it is very unlikely that faults occur because of short circuits in some of the equipment that has been mentioned previously [1]. However, the trans- mission lines being the element that covers most of the trajectory of the system and being subject to any type of climatic condition are more vulnerable and therefore are considered the most critical part of the power system and must be protected the best possible to keep them in excellent service conditions [2]. A quick detection of a fault event allows a quick restoration of the system, having as main objective the reduction of the interruption times of the power supply. The Fourier transform is an excellent tool used for the analysis of stationary signals. It has certain deficiencies, especially when you want to perform the analysis and detection of sudden changes that may be contained in those signals considered nonstationary. Currently there are a variety of sophisticated tools, which are very useful when performing fault analysis in transmission lines. That is why, in the present work, the use of the tool known as Wavelet transform is proposed to implement a methodology that is capable of detecting and identifying the fault event. Main objective of this paper is to apply a methodology to detection, classification and localization of faults that occur in transmission lines of electrical power systems, by simulating electromechanical perturbations in a test system and analysing the transients produced, using the wavelet transform and Support Vector Machine (SVM) classifier.

With the help of the MATLAB program, and the use of Wavelet transform tools, the signals obtained from the simulation in the test system for the identification of patterns are analyzed, with which it is possible to detect the possible types of faults caused by shorts. 


\section{Theoretical Framework}

An electrical power system (EPS) consists of several elements that are interconnected with each other. Regardless of the simplicity or complexity presented by the electrical networks, these are made up of a series of conventional elements that make their operation feasible. Among the main elements are the following:

- Generators

- Transformers

- Lines of transmission

- Loads

- Bars

Below is a brief description of the elements that will be used later to perform the modeling of the test system.

\subsection{Sources}

In general, the generator is a rotating machine that converts mechanical power into alternating electrical power. A source or generator corresponds to a synchronous machine. The primary function of a generator is the provision or supply of a fixed value of active power in such a way that the regulated voltage can be maintained in the bar in which it is connected by the injection of reactive power [3]. For the modeling of the ideal source a balanced three-phase source is used.

\subsection{Transmission Lines}

They are considered the most common element of an ESP, responsible for transmitting at great distances the energy generated in the different generation plants to the different consumption centers.

The study of transmission line models is of great importance, taking into account that thanks to them it is possible to understand the behaviour of different transient phenomena by means of mathematical models that are sufficiently accurate for a specific frequency range [4].

For the study proposed in the present work, the J. Marti transmission line model belonging to the LCC group of lines [5] is used The $\mathrm{J}$ Marti model is considered the most accurate for the analysis of transients since it considers parameters that are frequency dependent. Through rational functions, it performs an approximation of the characteristic impedance and the propagation constant. It uses a constant transformation matrix to convert values that are in the modal domain to the phasor domain. Taking into account the distributed nature of the losses and the dependence of the parameters, it is better to develop the line equations in the frequency domain [5] as shown in equations (1) and (2):

Where,

$$
\begin{aligned}
& V_{k}(\omega)=Z_{C}(\omega) I_{k}(\omega)+E_{m h}(\omega) \\
& V_{m}(\omega)=Z_{C}(\omega) I_{m}(\omega)+E_{k h}(\omega)
\end{aligned}
$$

$$
\begin{array}{r}
E_{m h}(\omega)=\left[V_{k}(\omega)+Z_{C}(\omega) I_{k}(\omega)\right] e^{-\gamma(\omega) l} \\
E_{k h}(\omega)=\left[V_{m}(\omega)+Z_{C}(\omega) I_{m}(\omega)\right] e^{-\gamma(\omega) l}(4)
\end{array}
$$

They are the sources of voltage at the nodes $k$ and $m$. The characteristic impedance $Z_{C}$ and the propagation constant $\gamma$ are given by equations (5) and (6) respectively:

$$
\begin{gathered}
Z_{C}(\omega)=\sqrt{\frac{R^{\prime}(\omega)+j \omega L^{\prime}(\omega)}{G^{\prime}(\omega)+j \omega C^{\prime}(\omega)}} \\
\gamma(\omega)=\sqrt{\left[R^{\prime}(\omega)+j \omega L^{\prime}(\omega)\right] \cdot\left[G^{\prime}(\omega)+j \omega C^{\prime}(\omega)\right]}(6)
\end{gathered}
$$

Transforming equations (1) and (2) of the frequency domain to the time domain, we obtain equations (7) and (8) as shown below:

$$
\begin{aligned}
& V_{k}(t)=Z_{C}(t) * i_{k}(t)+\left[V_{m}(t)+Z_{C} * i_{m}(t)\right] * a(t)(7) \\
& V_{m}(t)=Z_{C}(t) * i_{m}(t)+\left[V_{k}(t)+Z_{C} * i_{k}(t)\right] * a(t)(8)
\end{aligned}
$$

Where:

$$
a=\frac{1}{\sqrt{L^{\prime} C^{\prime}}}
$$

$i_{k}=$ Current in node $k$

$i_{m}=$ Current in node $m$

$l=$ Total length of the line in $[\mathrm{km}]$

$a=$ Velocity of propagation of the traveling wave

$L^{\prime}=$ Inductance per unit length $[\mathrm{H} / \mathrm{km}]$

$C^{\prime}=$ Capacitance per unit length $[\mathrm{F} / \mathrm{km}]$

$R^{\prime}=$ Resistance per unit length $[\mathrm{D} / \mathrm{km}]$

$G^{\prime}=$ Conductance per unit length $[\mathrm{S} / \mathrm{km}]$

The mathematical model of J. Marti presents a disadvantage and is that it is not a very accurate model when working with sections of very short lines and low frequencies, this is due to the inaccuracies that arise when calculations are made in the domain of the time [5], [5].

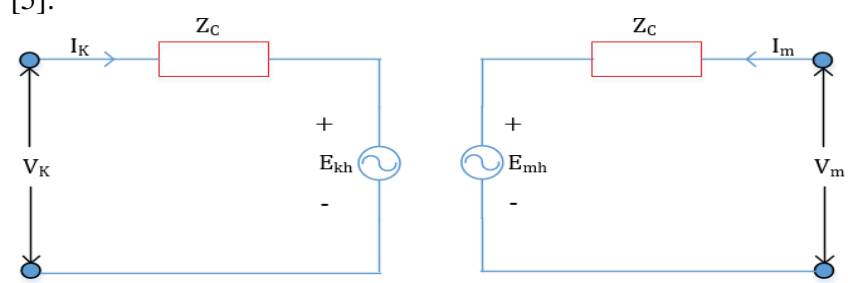

Fig. 1: Equivalent circuit of the J. Marti model in the frequency domain [5]

\subsection{Transmission Towers}

The transmission towers are metallic structures, whose purpose is to serve as support for the aerial conductors that make up the electric power transmission lines. To model the line of interest, a single-circuit transmission tower of $500 \mathrm{kV}$ will be used and for the rest of the lines that make up the test system, the typical structure used for $230 \mathrm{kV}$ transmission lines will be used.

\subsection{Transformers}

The function of the transformer, within the Electric Power System, is to raise or lower the voltage level according to the user's need. The model to be used is the hybrid transformer (Hybrid transformer), it is a model that was designed to cover the shortcomings of other existing models, it is a model capable of providing a response both in permanent regime and in transitory regime, This way it is possible to represent with a good precision the effects of the electric circuit and the magnetic circuit [6].

\subsection{Loads}

The load is the demanded power of the system by the final consumer [7]. Since the study is carried out in steady state, constant values are considered, both for $P$ and for $Q$. The values of $r(\Omega)$ and $X L(\Omega)$ can be calculated from the available data of $P, Q$ and $V$ using equations (10) and (11) respectively.

$$
\begin{gathered}
r=\left[\frac{\left(\frac{V \cdot 10^{3}}{\sqrt{3}}\right)^{2}}{\frac{P \cdot 10^{6}}{3}}\right] \\
X L=\left[\frac{\left(\frac{V \cdot 10^{3}}{\sqrt{3}}\right)^{2}}{\frac{Q \cdot 10^{6}}{3}}\right]
\end{gathered}
$$

\subsection{Bars}

The bars constitute the electrical nodes of any EPS, to which the different elements are connected: generators, transformers, loads, etc.

With, 


\section{Faults in Electrical Power Systems}

A fault can be defined as any event that interferes with the normal flow of current [8]. Generally, electrical power systems are exposed to the occurrence of any type of contingency, which may be external or internal [8].

Being understood by external contingencies those that are of environmental origin, as it is the case of atmospheric discharges, rays, climatic conditions like the humidity and the rain that affect the operation of devices like insulators, branches of trees that are put in contact with the lines, etc.

Meanwhile, internal contingencies occur during the operation of the system such as short circuits, over-voltages that are caused by switching effects in switches or accidental opening of disconnectors.

Short circuits are considered the most serious type of contingency, because during the existence of this type of events there is an excessive increase in the magnitudes of the currents of the phases involved, that is why in the shortest possible time these events must be cleared in order to avoid that the effects of the short circuit propagate to the rest of the system [8].

The short circuit is an electrical phenomenon that occurs when two or more points between which there is a potential difference are accidentally put in contact, as the main consequence there is an increase in the circulating currents up to the point at which said event occurred [9].

Because of the excessive increase in the magnitudes of the currents of the phases involved in the short circuit, several effects can occur [8] among which we can note the following:

- Joule effect: Produced by the heating of the conductors, it is a function of the magnitude and duration of the short-circuit current.

- Electrodynamic Efforts: The heating produced by the high short circuit currents can weaken the mechanical resistance, which could affect the deformation or rupture of the element in which the fault occurred.

- Voltage Variation: In those phases involved in the short circuit there is a voltage drop while, in the phases that are not involved there is an eventual increase in the voltage level.

- Interruption of the electrical supply: This occurs due to the opening of the electrical circuit by the devices associated with the protection system.

In an Electric Power System, one can find symmetrical (balanced faults) and asymmetric faults (unbalanced faults) [10].

The symmetrical or balanced faults are those in which the three phases are involved. Approximately in 5\% of the faults the 3 phases intervene, for this reason they are the least frequent [10].

Three Phase Fault: It originates when the three phases are put in contact in a same point of the system through a fault impedance [9]. Considered the most severe type of fault and at the same time is the least frequent.

Single-phase Earth Fault: It originates when any of the phases is put in contact with the earth of the system [8]. It is the fault that occurs most frequently in the transmission lines.

Biphasic Fault: This type of fault originates when two phases of the system come into contact [9].

Biphasic Earth Fault: This type of fault occurs when two phases come into contact with an external element that leads to earth [10].

\section{Protection for Transmission Lines}

The transmission lines to be exposed to the weather and therefore to any type of weather condition are the element in which the highest percentage of faults occur.

Once any fault has occurred regardless of the point at which said event occurred, the primary function of the protection system is to reduce the effects of short circuits and thus disconnect the circuit that is in fault as quickly as possible, so that the fault can be prevented from spreading to the rest of the system [11].

\subsection{Characteristics of Protection Systems}

For the protection system to operate properly, it is necessary that the operation of the system be linked to the following criteria [12]. Dependability: Degree of certainty in which the protection system will operate correctly in case of faults in its protection zone.

Sensitivity: Related to minimum values (current, voltage, frequency, etc.) at which the operation of the relay occurs. The protection system must have the capacity to unequivocally distinguish situations of fault from those that are not.

Speed: Related to the time required to issue the firing order. Since a fault must be cleared as quickly as possible in order to continue the service and prevent the rest of the system from collapsing.

Security: Degree of certainty in which the protection system will not operate in the event that faults occur outside its protection zone.

Selectivity: Capacity that the protection must have to identify if said event has occurred inside or outside its protection zone.

\subsection{Basic Components of a Protection System}

Among the main elements that make up a protection system are the following [9]:

Measuring Transformers: They allow obtaining information about the conditions to which the power system operates, they are in charge of registering and transforming the high values of current and voltage that circulate along the transmission line in standardized values for their later application to the relay. They are used to isolate the secondary circuits (control, protection and measurement equipment) from the high voltages present in the primary circuits.

Relays: They are the most important elements in the protection system because they are in charge of receiving and processing the information coming from the voltage transformers and current. They are devices designed to discriminate between normal and abnormal operating conditions of the system.

Switches or Circuit Breakers: These are the elements in charge of receiving the trip order from the relay, they operate under the control of the protection and their opening, coordinated with that of other switches, allows isolating the zone in which the fault occurred.

\subsection{Classes of Protections}

Main Protection: It is responsible for clearing the fault in the first instance. Disconnect the least number of elements to isolate the fault from the rest of the system. Once the fault has occurred, only the switches associated with that protection must be triggered to clear the fault [10].

The main protection divides the SEP into the so-called protection zones, with the protection area being understood as the portion of an electrical system protected by a protection system [9].

Backup Protection: It is the protection called to perform the clearance of the fault in the second instance [9]. Backup protection generally disconnects a larger portion of the system than the primary one. It is necessary to install this type of protection taking into account the following reasons that are described below:

- In case the main protection does not operate properly, the backup protection will be in charge of clearing the fault.

- $\quad$ Protect those areas of the power system, which are not protected by the main protection, due to the location of the measurement transformers.

- There is also local backup protection, which is installed in the same substation where the main protection is located, while the remote backup protection is that which 
is located in the substation adjacent to the one installed the main protection [10].

\subsection{Method of Protection of Transmission Lines}

There are different methods to protect the transmission lines, however, in the present work a brief review is made on distance protection since it is the most used method for the protection of transmission lines.

Distance Protection: For its operation, it requires current and voltage signals at its inputs [9]. The principle of operation of this relay is based on the calculation of the impedance between the relay and the point at which the fault occurred, relating the magnitudes of voltage and current coming from the measuring instruments. They work when the ratio is less than its pre-established value [12].

Depending on the topology of the power system it is possible to configure several distance operation zones, each zone with an appropriate operating time of coordination with the relays of the adjacent lines.

Among the main advantages of this type of protection is:

- They have greater sensitivity.

- They have a greater area of instantaneous operation.

- Greater ease of coordination and adjustment.

They are not affected by changes in the System's topology.

\section{Proposed Methodology}

\subsection{Data Reduction}

The data reduction techniques are applied to achieve a reduced representation of the original data set, it is much smaller in volume, and however, it closely maintains the integrity of the original data. For this reason, in this paper as a data reduction technique, the Discrete Wavelet Transform is used for extracting the high frequency components (detail coefficients) of the signal of interest. The mother wavelet employed is the Daubechies $4(\mathrm{db} 4)^{\prime}$, since it presents a good performance in the analysis of faults in power systems [13]. This type of wavelet adapts very well when it comes to identifying high frequency transients for short periods of time, as well as the behavior of low frequency transients for longer periods of time [14].

The decomposition of the signals of interest is carried out using the MATLAB programwith the help of the command "wavedec".

The "wavedec" command performs a multilevel wavelet decomposition in a single dimension (1-D), for which it uses a specific wave type "wname" or a specific set of Wavelet decomposition filters.

$[\mathrm{C}, \mathrm{L}]=$ wavedec $(\mathrm{X}, \mathrm{N}$, 'wname') returns the wavelet decomposition of the $\mathrm{X}$ signal, for a decomposition level $\mathrm{N}$, where $\mathrm{N}$ is a strictly positive integer value and using a specific Wavelet type "wname"

\subsection{Detection of Faults}

According to [13], [15] for the detection of faults, the proposed method requires the following steps:

1. Calculate the positive sequence current from the phase currents obtained in the different simulations carried out.

2. Decompose the positive sequence current signal using the DWT to obtain the detail coefficients at level $\mathrm{N}=1$.

3. Calculation of the spectral energy from the detail coefficients obtained from performing the decomposition of the signal using DWT.

The spectral energy can be calculated from equation (12):

$$
S E^{j}=\sum_{k=1}^{\frac{N_{W}}{2^{j}}}\left[D_{j}(k)\right]^{2} ; \quad k=1,2,3, \ldots, \frac{N_{w}}{2^{j}}
$$

Where,

$D_{j}(k)$ : Is the detailed coefficient for a level $j$.
$N_{w}$ : Size of the window (number of samples in a cycle of the original signal).

The condition to consider the existence of a fault is that there is a change in the spectral energy of about five times the spectral energy before the occurrence of the fault.

Where:

$$
S E_{1}>5 * S E_{N C}
$$

$S E_{N C}$ : Spectral energy at normal conditions.

$S E_{1}$ : Spectral energy once the fault occurs.

In Figure 2, one can see the spectral energy, represented by the magenta line. Meanwhile, the segmented red line represents the threshold value. The spectral energy at normal conditions has a value of $S E_{N C}=0.042$

According to [13] the value of the spectral energy at normal conditions is multiplied by five in order to establish the value known as threshold and with which it will be possible to discriminate situations of existence or not of fault.

$$
\text { Threshold }=5 * 0.042=0.21
$$

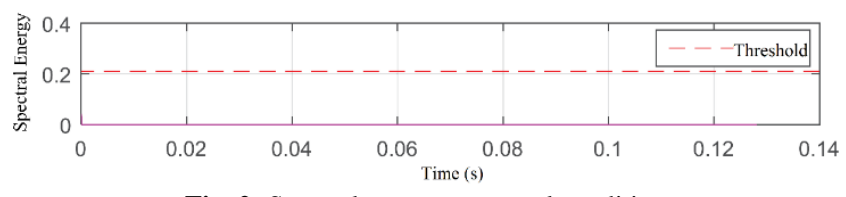

Fig. 2: Spectral energy at normal conditions

In case that the condition of equation (13) is met, the algorithm will discriminate a fault situation, otherwise it will discriminate the non-existence of fault. The flow diagram for fault detection is illustrated in Figure 3.

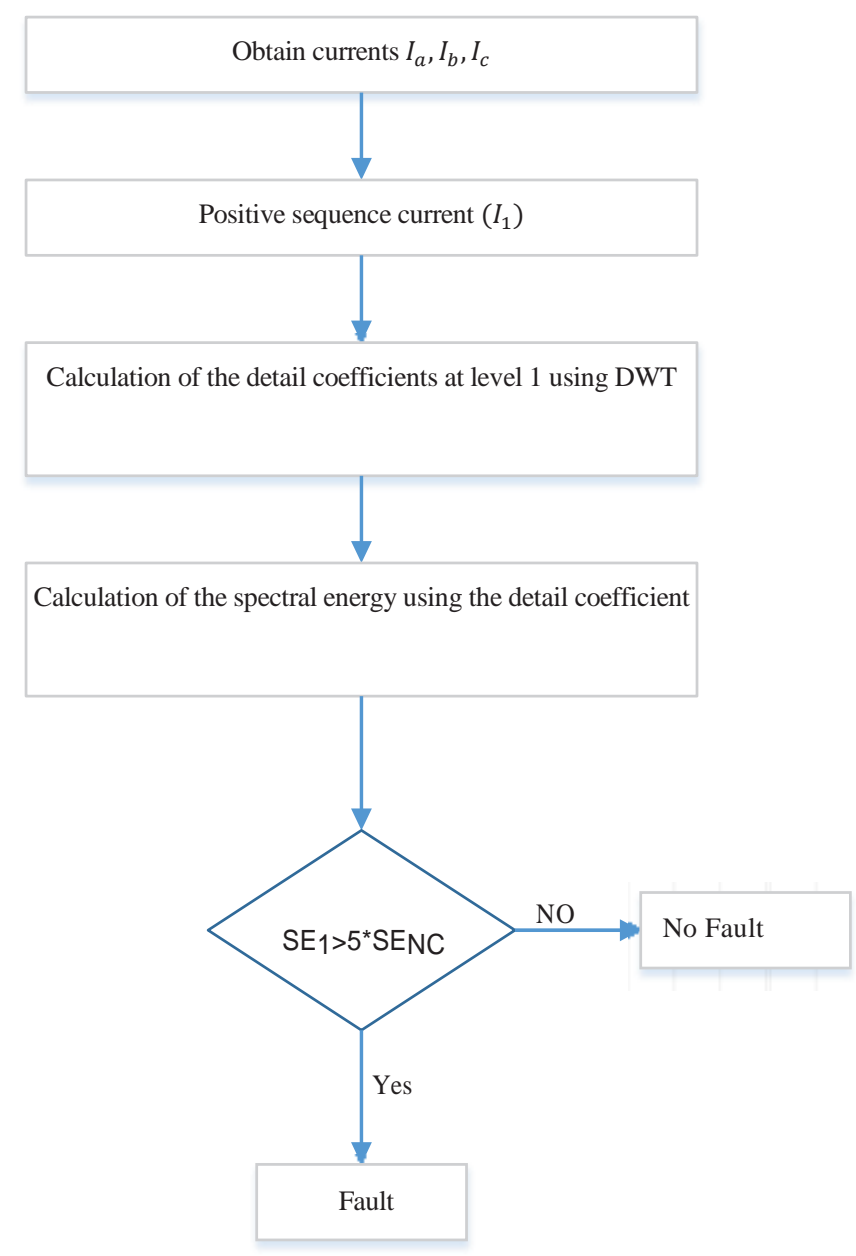

Fig. 3: Flow diagram for fault detection 


\subsection{Identification and Classification of Faults}

The proposed method consists of obtaining a model, with which the type of fault can be identified and classified.

According to the following procedure and with the help of MATLAB, the model is obtained:

Obtaining the Input Data Matrix: The data matrix Q is formed from the samples obtained through the Wavelet decomposition of each of the signals, both current and voltage obtained in the different simulations for each kilometre of the transmission line.

The decomposition of each of the signals is done to obtain the detail coefficients for a level 7 using the Daubechies mother wavelet $4(\mathrm{db} 4)$. The signals obtained from the different simulations present total of 12800 samples, which when analyzed using the discrete wavelet transform for a level $\mathrm{N}=7$, signals with a total of 106 samples are obtained.

Figure 4 shows an example corresponding to current signals obtained in the simulation of a single-phase fault, each of the signals with a total of 12800 samples.

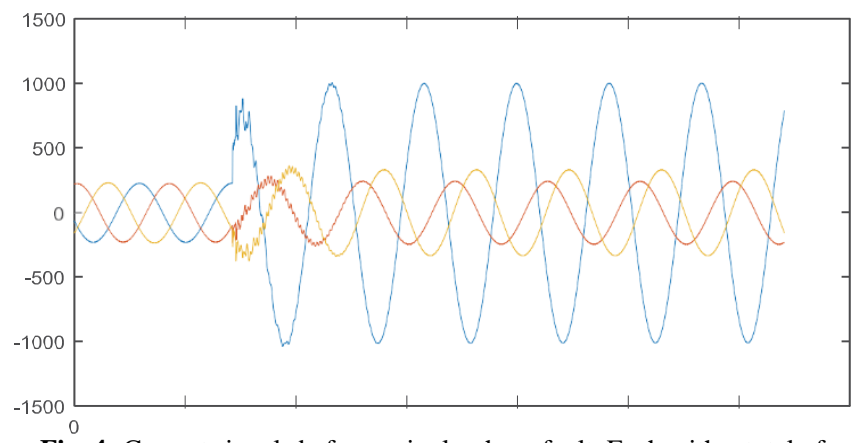

Fig. 4: Current signals before a single-phase fault. Each with a total of 12800 samples

Figure 4 shows the decomposition corresponding to current signals at a level 7 and using the Daubechies mother wavelet 4 (db4), each of the signals with a total of 106 samples.

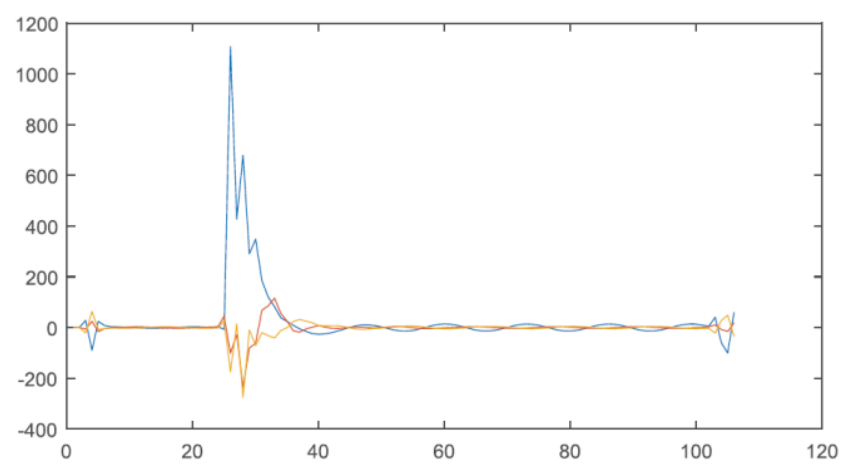

Fig. 5: Decomposition of current signals using DWT. Each with a total of 106 samples

In this paper, two input matrices are used, the first formed only by the detail coefficients obtained from the wavelet decomposition of the current signals and the second formed by the detail coefficients obtained from the wavelet decomposition of the voltage signals.

Once the decomposition has been completed for the different signals of interest, the first input matrix is formed, in this case, as already mentioned, the matrix is formed only by the detail coefficients obtained from the wavelet decomposition of the current signals measured in bar 1 .

For the two matrices shown above, the concept of normalization applies. In the second case, the matrix is composed of two types of different signals, which implies that both the units of the currents $[\mathrm{kA}]$ and the units of the voltages $[\mathrm{kV}]$ must be taken into account. Normalization: It consists in transforming all the values of the input matrix, in such a way that they are comparatively similar
[16], [17]. When talking about data normalization, one of the typical intervals is $[-1,1]$.

For normalization the concept of Min-Max Normalization [16] is used, which performs a linear transformation in the original data, in this case the $\mathrm{Q}$ matrix in the specified interval $\left[\right.$ new_min $A$, new_max $\left.\max _{A}\right]$ i.e. the interval of $[-1,1]$.

Let $Q=\left[q_{1}, q_{2}, . ., q_{i}, ., q_{n}\right]$ a data vector with $n$ elements, where its minimum and maximum values are given by:

$$
\begin{aligned}
\min _{Q} & =\min \left\{q_{1}, q_{2}, . ., q_{i}, . ., q_{n}\right\} \\
\max _{Q} & =\max \left\{q_{1}, q_{2}, . ., q_{i}, ., q_{n}\right\}
\end{aligned}
$$

Where the elements of the normalized vector $Q_{i}^{\prime}=$ $\left[q_{1}^{\prime}, q_{2}^{\prime}, ., q_{i}^{\prime}, ., q_{n}^{\prime}\right]$ in the interval of $[-1,1]$ are calculated from the expression of equation (16):

$$
Q_{i}^{\prime}=\frac{Q_{i}-\min _{Q}}{\max _{Q}-\min _{Q}} \cdot\left(\text { new }_{\min _{A}}-\text { new }_{\text {max }_{A}}\right)+\text { new_ }_{-} \max _{A}(16)
$$

Where $Q_{i}$ corresponds to the value of the sample $i$.

\subsection{Fault Location using SVM}

For the location of the faults, the method of the traveling waves was used, extracting the relevant information through the WT and having as support in the identification, Support Vector Machine classifier is used. In the case of the present work, the information of the components of high frequency (traveling wave), obtained from the first level of detail of the wavelet transform.

Basics of SVM: Support Vector Machines were proposed by Vapnik and Lerner as an automatic learning system based on the theory of statistical learning [17], [18], [19]. They are models whose dependence is not based on a specific probability distribution and within the framework of the statistical learning theory they have the ability to learn from a set of experimental data or training data that are commonly taken from the real environment.

The idea is based on the classic concept of optimal separation hyperplane, where the separation vector is expressed as a function of training samples. Likewise, the theory of statistical learning incorporates a series of aspects that give the SVM the generalization capacity superior to that of other learning methods [20]. The ability to generalize is mainly a consequence of margin maximization.

The SVM have been consolidated as a tool that has attracted the attention of researchers in the area of automatic learning and pattern recognition. Understanding by classification of patterns to the task of positioning an object within each of the categories called classes, for which, is part of a set of patterns that are associated with each object. The term "pattern" is used to indicate a vector of data $x$ of dimension $n$, where $x=\left(x_{1}, ., X_{n}\right)$ where the components $x_{i}$ represent the measurements of the characteristics of an object [21]. Usually these characteristics which are variables detailed by the researcher have a significant weight in the classification results.

Hyperplane of Optimal Separation: Tool used as separation boundary between two classes considered, in such a way that the distance between training data is maximized.

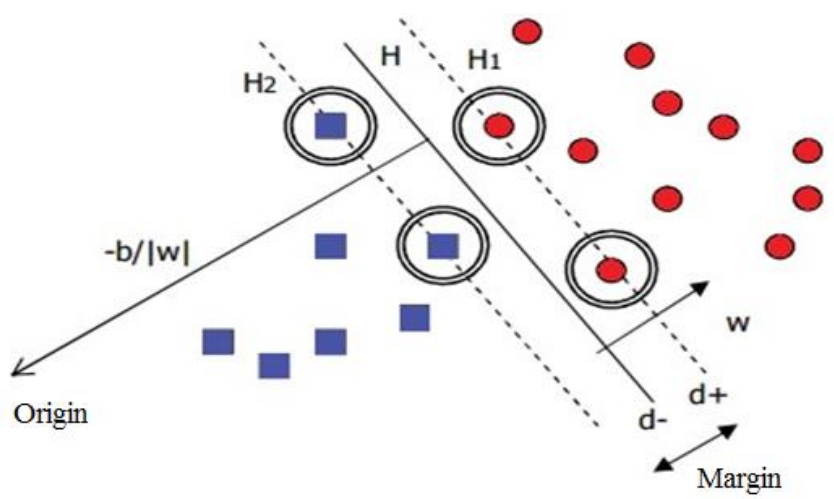

Fig. 6: Selection of the optimal separation hyperplane [21] 
The hyperplane $H$, thus obtained is called the optimal separation hyperplane, for which the margin is maximum. The margin is obtained as the distance between the two hyperplanes, $H_{1}$ and $H_{2}$, which contain at least one point of each class, these points are known as support vectors.

Without the points enclosed in a circle (Figure 6), called support vectors the solution space found would be different.

Use of SVMs in the MATLAB Environment: Taking into account the advantages and facilities for matrix calculation and the different tools related to machine learning, in this case the Support Vector Machines (SVM's), in this work MATLAB will be used together with the tool "Statistical and Machine Learning Toolbox " 'and by means of the command' 'fitcecoc' 'to carry out the process of training and validation of the model.

$\mathrm{Mdl}=$ fitcecoc $(\mathrm{Q}, \mathrm{Y})$ adjusts $\mathrm{k}(\mathrm{k}-1) / 2$ binary classifiers for Vector Support Machine models using the "one on one" combination scheme, additionally as we have data that are not linearly separable in the input space, the data is transformed, for this the command in use uses by default the type of Gaussian kernel that, as already mentioned above, is a general purpose Kernel function mostly used in classification processes.

$\mathrm{Q}$ is the matrix that contains only predictive variables of numerical type with $\mathrm{N}$ number of rows and $\mathrm{P}$ number of columns. In contrast, $\mathrm{Y}$ is the response matrix that contains $\mathrm{N}$ number of labels. And it has $\mathrm{K}$ classes, the matrix Y can be any of the following:

- It can be a categorical matrix, a logical vector, a numerical vector, or a matrix formed by strings of characters.

- The returned model Mdl is a Classification ECOC class object. (ECOC): Error-correcting output code model.

- Classification ECOC is a model that reduces the problem of classifying three or more classes to a set of binary classifiers.

- The configuration used by the mentioned command is the following: $\mathrm{Mdl}=$ fitcecoc $(\mathrm{Q}, \mathrm{Y})$

Taking into account what has been described above, we proceed with the selection of the data that will be used, both for the training and for the validation of the model.

\section{Simulation Results}

\subsection{Simulation Parameters}

The cases considered for the evaluation of the algorithm, for the particular system previously described, were the following:

1. Type of fault: The eleven types of possible faults (AG, $\mathrm{BG}, \mathrm{CG}, \mathrm{ABC}, \mathrm{BCG}, \mathrm{CAG}, \mathrm{AB}, \mathrm{BC}, \mathrm{CA}, \mathrm{ABC}$ and ABCG) were considered.

2. Fault impedance (resistive only - Rf): $100 \Omega, 300 \Omega$, $500 \Omega$.

3. Fault timing: $0.23 \mathrm{sec}$.

4. Fault location: 5 to $195 \mathrm{~km}$.

\subsection{Results}

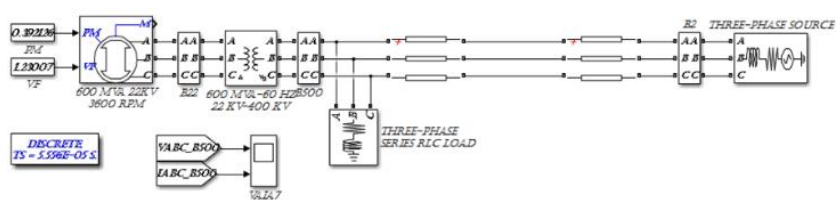

Fig. 7: Simulink model without fault

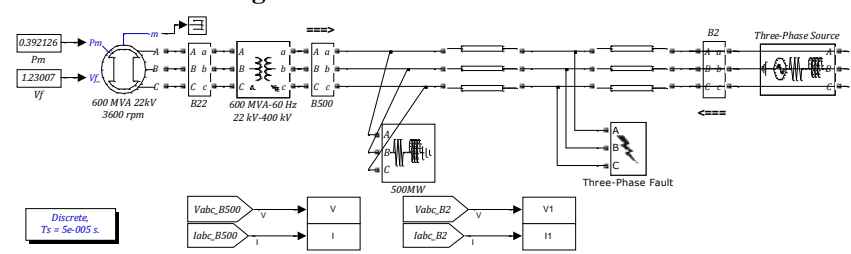

Fig. 8: Simulink model considered for the evaluation of the proposed system

Three Phase Voltage

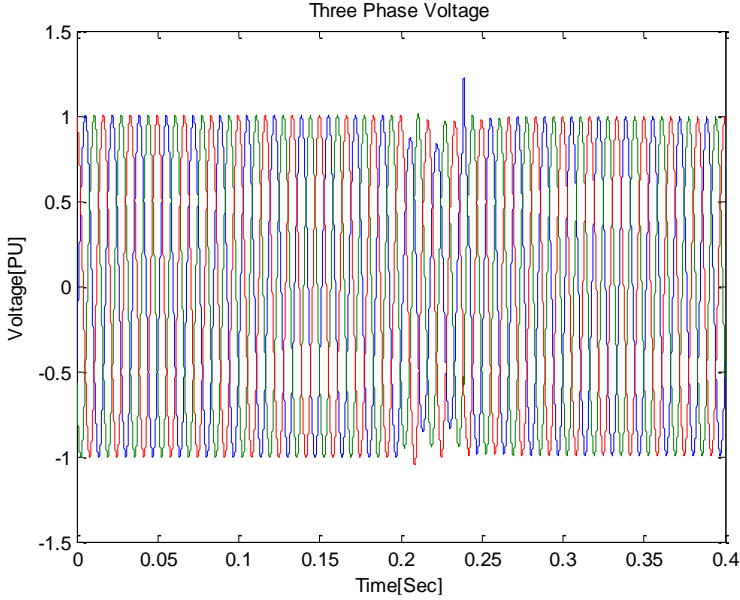

Fig. 9: Voltage response of three phase circuit

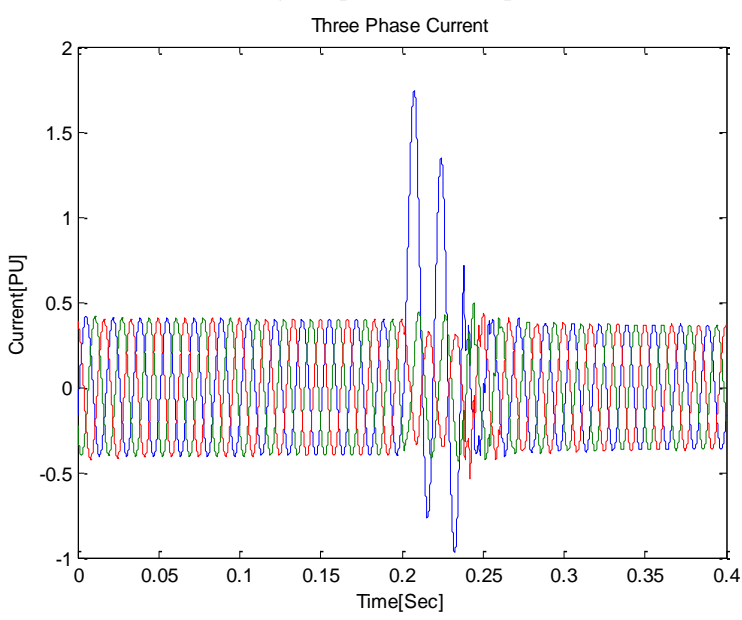

Fig. 10: ABC fault in three phase circuit
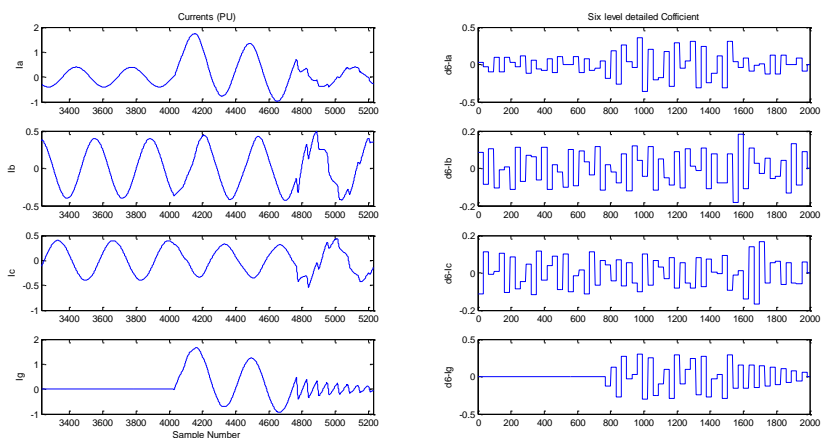

Fig. 11: Detailed coefficient for various faults

\section{Conclusion}

An alternative method has been applied for the detection of faults caused by short circuits along a transmission line of an electrical power system, through the application of algorithms implemented in the MATLAB platform. The algorithm uses the tool known as the Discrete Wavelet Transform for the analysis of transients and the tool known as Support Vector Machines to obtain a model with which it is possible to identify and classify the different types of faults. Regarding the detection of faults, the prototype or mother wavelet function used was the Daubechies with 4 fading moments (db4), the same one that according to the obtained results turned out to be adequate for the different cases analyzed in the present work. One of the main advantages of carrying out the normalization process of the database with which you want to work is that you obtain comparatively similar data within a select- 
ed normalization interval, this translates into computational savings, specifically when performs training to obtain different models.

The tool used for the automatic simulation of faults is a simulation strategy that allows obtaining the different signals of interest automatically and systematically, considerably reducing the simulation time in the different cases studied. The inclusion of sophisticated tools in terms of the implementation of new methodologies in the area of electrical protection has contributed to the development of a great variety of algorithms that allow detecting, classifying and locating the fault in a fast, reliable and precise way.

\section{References}

[1] Koley, E., Verma, K. and Ghosh, S., 2015. An improved fault detection classification and location scheme based on wavelet transform and artificial neural network for six phase transmission line using single end data only. SpringerPlus, 4(1), p.551.

[2] Chui, C.K., 2016. An introduction to wavelets. Elsevier.

[3] Eldin, A.H. and Refaey, M.A., 2011. A novel algorithm for discrimination between inrush current and internal faults in power transformer differential protection based on discrete wavele transform. Electric Power Systems Research, 81(1), pp.19-24.

[4] Cheong, W.J. and Aggarwal, R.K., 2002, October. Accurate fault location in high voltage transmission systems comprising an improved thyristor controlled series capacitor model using wavelet transforms and neural network. In Transmission and Distribution Conference and Exhibition 2002: Asia Pacific. IEEE/PES (Vol. 2, pp. 840-845). IEEE.

[5] Marti, J.R., 1982. Accuarte modelling of frequency-dependent transmission lines in electromagnetic transient simulations. IEEE transactions on power apparatus and systems, (1), pp.147-157.

[6] Chiesa, N., Mork, B.A. and Høidalen, H.K., 2010. Transformer model for inrush current calculations: Simulations, measurements and sensitivity analysis. IEEE Transactions on Power Delivery, 25(4), pp.2599-2608

[7] Schettler, F., Huang, H. and Christl, N., 2000. HVDC transmission systems using voltage sourced converters design and applications. In Power Engineering Society Summer Meeting, 2000. IEEE (Vol. 2, pp. 715-720). IEEE.

[8] Grainger, J.J. and Stevenson, W.D., 1994. Power system analysis (Vol. 621). New York: McGraw-Hill.

[9] Sleva, A.M., 2009. Protective relay principles. CRC Press.

[10] Kothari, D.P. and Nagrath, I.J., 2011. Modern power system analysis. Tata McGraw-Hill Education.

[11] Anderson, P.M., 1998. Power system protection. Wiley.

[12] Elmore, W.A., 2003. Protective relaying: theory and applications (Vol. 1). CRC press.

[13] Sawatpipat, P. and Tayjasanant, T., 2010, May. Fault classification for Thailand's transmission lines based on discrete wavelet transform. In Electrical Engineering/Electronics Computer Tele communications and Information Technology (ECTI-CON), 2010 International Conference on (pp. 636-640). IEEE.

[14] Kim, C.H. and Aggarwal, R., 2000. Wavelet transforms in power systems. Part 1: General introduction to the wavelet transforms. Power Engineering Journal, 14(2), pp.81-87.

[15] Makming, P., Bunjongjit, S., Kunakorn, A., Jiriwibhakorn, S and Kando, M., 2002, October. Fault diagnosis in transmission lines using wavelet transform analysis. In Transmission and Dis tribution Conference and Exhibition 2002: Asia Pacific. IEEE/PES (Vol. 3, pp. 2246-2250). IEEE

[16] Han, J., Pei, J. and Kamber, M., 2011. Data mining: concepts and techniques. Elsevier.

[17] Kantardzic, M., 2011. Data mining: concepts, models, methods, and algorithms. John Wiley \& Sons.

[18] Cristianini, N. and Shawe-Taylor, J., 2000. An introduction to support vector machines and other kernel-based learning methods. Cambridge university press.

[19] Ekici, S., 2012. Support Vector Machines for classification and locating faults on transmission lines. Applied Soft Computing, 12(6), pp.1650-1658.

[20] Chen, K., Huang, C. and He, J., 2016. Fault detection, classification and location for transmission lines and distribution systems: a review on the methods. High voltage, 1(1), pp.25-33.

[21] Rautaray, S.S. and Agrawal, A., 2015. Vision based hand gesture recognition for human computer interaction: a survey. Artificial Intelligence Review, 43(1), pp.1-54. 